\title{
Caracterización de las actividades interdisciplinarias en una facultad de artes Colombiana
}

\section{Characterization of interdisciplinary activities in a Colombian faculty of arts}

\author{
Alejandro Antonio Naranjo-Gaviria' \\ Elisabeth Herreño-Téllez ${ }^{2}$
}

Recibido: marzo 07 de 2019 Aceptado: noviembre 18 de 2019

\begin{abstract}
Resumen
La fragmentación del conocimiento distancia a las organizaciones educativas de la realidad y pone en entredicho la legitimidad de la academia ante la sociedad. Esta investigación busca contribuir en la resolución de problemas asociados a la gestión del conocimiento y el trabajo interdisciplinar en una Facultad de Artes. El estudio fue de tipo exploratorio y descriptivo, analizó el conocimiento implícito y explícito de los docentes mediante la aplicación de una encuesta estructurada y entrevistas realizadas a los docentes. La muestra estuvo conformada por 22 docentes de una universidad colombiana. Se encontró que existe un potencial de interacción entre los integrantes del equipo que puede ser explorado en el desarrollo de proyectos, para lo cual es necesario enfocar la visión y generar espacios para la construcción colectiva de proyectos desde una perspectiva sistémica.
\end{abstract}

Palabras clave: gestión de conocimiento, proyectos interdisciplinarios, facultad de artes, educación universitaria.

\begin{abstract}
Knowledge fragmentation separates educational organizations from reality and calls into question the legitimacy of the academic endeavors before society. This research seeks to contribute to the resolution of knowledge management problems associated with interdisciplinary work in a faculty of arts. The exploratory and descriptive study analyzes the implicit and explicit knowledge of faculty professors through the use of interviews and structured surveys. The sample was formed by 22 teachers of a Colombian university. The results show a potential interaction between team members of the faculty, suitable for exploration in project development, for which it is necessary to focus on a vision and create spaces for collective project construction from a systemic perspective.
\end{abstract}

Keywords: knowledge management, interdisciplinary projects, faculty of arts, higher education.

1 Arquitecto, Magíster en Arquitectura y Urbanismo, Universidad de San Buenaventura, Medellín, Colombia. E-mail: alejandro.naranjo@usbmed. edu.co. Orcid: 0000-0002-1856-4467

2 Diseñadora Industrial, Magíster en Ingeniería, Universidad de San Buenaventura, Medellín, Colombia. E-mail: elisabeth.herreno@usbmed.edu.co Orcid: 0000-0003-0205-7767 


\section{Introducción}

El presente artículo busca caracterizar el trabajo interdisciplinar en la Facultad de Artes Integradas (FAI), de la Universidad de San Buenaventura, seccional Medellín, Colombia, como insumo para dinamizar iniciativas de este tipo. Con el fin de generar contrastes y establecer puentes entre el contexto institucional y antecedentes en el tema, se hace una revisión de aspectos claves en la gestión del conocimiento, tales como: los tipos, áreas, generación, la dinamización del conocimiento, entre otros (Kuhn, 2004; Maturana \& Varela, 2003; Morin, 2007; Nonaka \& Takeuchi, 1995; Colciencias, 2018a; Thackara, 2012). Así mismo, para el desarrollo de proyectos interdisciplinarios, se analizaron: los conceptos asociados, las razones, los problemas y alternativas para resolverlos, así como las formas de evaluación (Bark, Kragt \& Robson, 2016; Carr, Loucks \& Blöschl, 2018; Rijnsoever \& Hessels, 2011; Thompson, 2014).

La evidencia personal de los autores al interior de la $\mathrm{FAl}$, es que entre los docentes no se generan dinámicas que permitan o faciliten el trabajo en equipo, situación que se evidencia en proyectos desarrollados por un solo docente, en temáticas disciplinarias muy limitadas. Estas dificultades se presentan por la falta de conocimiento sobre los campos de trabajo de los compañeros, así como por miedo a la interacción con otros, pocos espacios de relación y en algunos casos por egocentrismo. La presente caracterización busca contribuir en la dinamización del conocimiento, fortaleciendo el trabajo colaborativo y en red de los diferentes actores, para la consolidación de procesos innovadores, como lo enuncian Galeano-Barrera et al. (2018), en torno a las actividades de la FAI en proyectos interdisciplinarios que den respuesta a las necesidades del medio.

Pese a que la variedad, densidad y reciprocidad son factores de éxito en una economía interactiva, las organizaciones dentro de las que vivimos: "encapsulan el conocimiento y no le dan salida hacia aquellos contextos en los cuales es requerido
(Thackara, 2012)". De acuerdo con Morin (2007), la gran dificultad en las organizaciones se debe a las formas de estructurar el conocimiento, simplificándolo o hiperespecializándolo, lo cual hace que se fragmente y aleje de la realidad. Por su parte, el Papa Francisco I (2015), establece que "los conocimientos fragmentarios y aislados pueden convertirse en una forma de ignorancia, si se resisten a integrarse en una visión más amplia de la realidad".

Para que una organización pueda desarrollarse, es necesario dinamizar las capacidades, hechos e información que tienen los individuos para el abordaje de situaciones cotidianas a las que se enfrentan (Morin, 2007). Al mismo tiempo, es necesario que los sujetos interactúen con el contexto (Maturana \& Varela, 2003), escudriñando "nuevos terrenos para encontrar inspiración y cultivar el hábito de buscar personas, lugares, organizaciones, proyectos e ideas" (Thackara, 2012). En este contexto, el trabajo interdisciplinar plantea posibilidades a través de las cuales se pueden encarar estos retos. A continuación, se identifican aspectos claves relacionados con el concepto de interdisciplinariedad, agrupándolos en las siguientes categorías de análisis: razones, aproximaciones al término, problemas asociados, dinamización del conocimiento para la resolución de problemas y evaluación de la interdisciplinariedad.

\section{Marco teórico y metodología}

\subsection{Razones para la interdisciplinariedad}

La necesidad de trascender el conocimiento, resolver problemas y transgredir el statu quo, representan las principales motivaciones que han potenciado el desarrollo del trabajo interdisciplinario (Thompson, 2014). En el caso de la trascendencia, la interdisciplinariedad ha permitido darle coherencia a lo construido por la humanidad a lo largo de su historia, generando síntesis en una época de creciente fragmentación del conocimiento y la cultura. Por su parte, la necesidad de abordar problemas difíciles de resolver, ha llevado a combinar fortalezas, métodos, recursos 
y desarrollos procedentes del conocimiento disciplinar. Finalmente, el sistema de conocimiento ha recurrido a la interdisciplinariedad, con el fin de poder generar transgresiones, encontrar posibilidades e integrar otras maneras de producir conocimiento.

De acuerdo con Rijnsoever y Hessels (2011), entre las razones que existen para participar en investigaciones interdisciplinares, se encuentran:

(...) Acceso a experiencia e instrumentos, fertilización cruzada entre disciplinas, acceso a financiación, obtención de prestigio o visibilidad, aprendizaje de conocimientos tácitos sobre una técnica, mejora de la productividad, diversión y placer. (p. 464).

La posibilidad de integrar investigadores en las interfaces y fronteras de sus respectivas disciplinas, permite abordar tareas que se encuentran en los límites y espacios entre sistemas y subsistemas, las cuales requieren la colaboración entre diversos actores; incluso al cruzar estas fronteras, se pueden formar nuevas disciplinas (Rijnsoever \& Hessels, 2011; Doucet \& Janssens, 2011).

La interdisciplinariedad representa una alternativa, a través de la cual se puede combatir la lentitud de la academia para moverse o para que sus resultados impacten otros sectores de la sociedad, lo cual redunda en la legitimidad de esta ante la sociedad (Thompson, 2014). Así mismo, puede imprimir dinámicas que motivan a sus integrantes, gracias a la posibilidad de trabajar con nuevos colegas de disciplinas diferentes (Bark, Kragt \& Robson, 2016).

\subsection{Aproximaciones al término de interdisciplinariedad}

Múltiples han sido las discusiones y la creación de organizaciones en torno al tema, desde que en 1970 se realizó el 1er. seminario en interdisciplinariedad, copatrocinado por la Organización para la Cooperación y el Desarrollo Económico, OECD (Thompson, 2014). Inicialmente, la interdisciplinariedad se definió como:

(...) La interacción entre 2 o más disciplinas diferentes. La interacción puede abarcar desde la simple comunicación de ideas hasta la integración mutua de conceptos organizativos, metodología, procedimientos, epistemología, terminología, datos y organización de la investigación y la educación en un campo bastante grande (OECD, 1972, (p. 24).

No obstante, varios son los términos asociados a este concepto y es necesario tener claridad en los límites de unos y otros. Tal como lo proponen Bark et al. (2016), dependiendo de la manera como se integran diferentes disciplinas, varía la manera como puede clasificarse una investigación, ver figura 1.

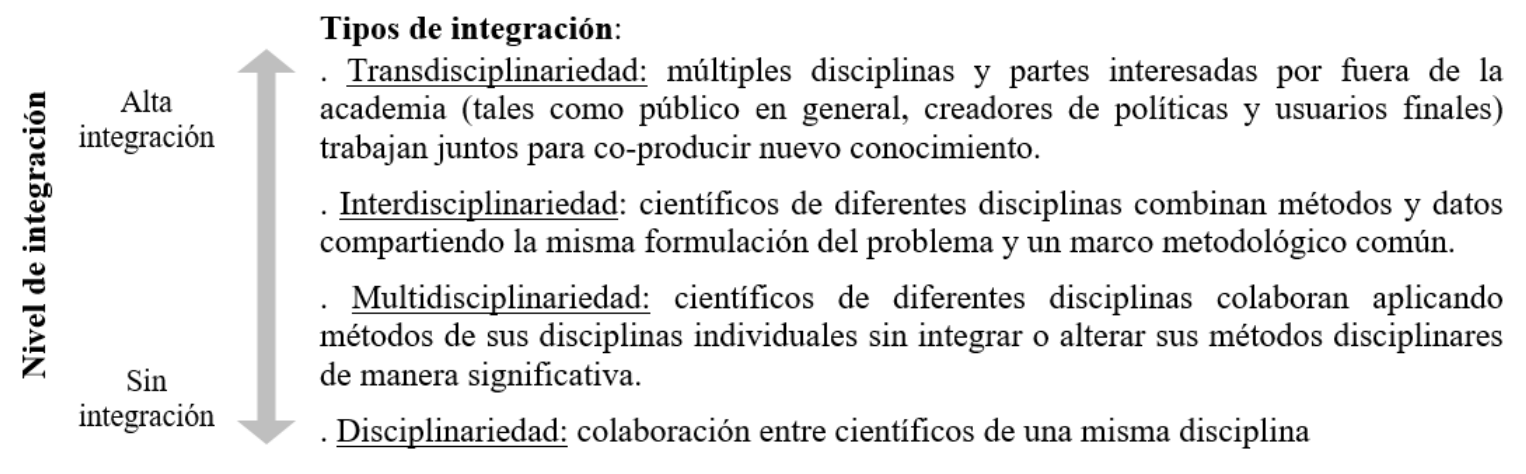

Figura 1. Tipos de integración entre las disciplinas (adaptado de Bark et al., 2016). 
De manera complementaria al concepto de interdisciplinariedad, Rijnsoever \& Hessels (2011) proponen que esta:

(...) Se refiere a la integración o síntesis de dos o más disciplinas, cuerpos de conocimiento o modos de pensamiento diferentes para producir un significado, obtener una explicación o un producto más extenso y poderoso que sus partes constituyentes. (p. 464).

Asimismo, Thompson (2014), identifica en el concepto de transdisciplinariedad: una posibilidad para evolucionar de un conocimiento científico confiable hacia el conocimiento socialmente robusto, que trasciende la dicotomía del experto / mundano, gracias a la promoción de nuevas alianzas entre la academia y la sociedad. De acuerdo a lo propuesto por Kuhn (2004), mientras que los conceptos de "disciplinariedad" pueden circunscribirse al "modo 1" del modelo de producción del conocimiento, caracterizado por estructuras jerárquicas, homogéneas y delimitadas a una disciplina en particular; la transdisciplinariedad se encuadra dentro del "modo 2", asociado a conceptos de complejidad, no linealidad y heterogeneidad.

\subsection{Problemas asociados a la interdisciplinariedad}

Pese a las posibilidades que se derivan del trabajo interdisciplinar, participar en proyectos de esta naturaleza trae consigo desafíos de comunicación entre disciplinas, falta de claridad en torno a los objetivos y dificultades para integrar diferentes métodos disciplinares (Bark et al., 2016). En este contexto, quienes tienen a su cargo funciones técnicas (ejecutores), pueden experimentar falta de control y comprensión por parte de la dirección general del proyecto (organizadores); en tal situación, las tareas que se les pide realizar a los primeros, parecen desconectadas de la generalidad, lo que a su vez afecta la disposición para trabajar.

Así mismo, la estructura de las instituciones educativas y de investigación tiende a estar organizada en torno a disciplinas y sus procedimientos de promoción y permanencia, se basan en la excelencia de una sola disciplina. Esto también se refleja al momento de publicar, puesto que "en promedio, las publicaciones interdisciplinarias reciben menos citaciones que las monodisciplinarias" (Rijnsoever \& Hessels, 2011). De otra parte, "estimular la investigación interdisciplinaria puede (...) disminuir las recompensas de las contribuciones monodisciplinarias, las cuales son cruciales para el desarrollo de campos científicos particulares" (Rijnsoever \& Hessels, 2011). Adicionalmente, el proceso de gestión propio de un proyecto interdisciplinario, genera estrés y reduce el tiempo que se podría destinar a la investigación disciplinar (Bark et al., 2016).

2.4 Dinamización del conocimiento para la resolución de problemas

Para dinamizar una organización, es fundamental identificar el conocimiento dentro de la misma, lo que Nonaka y Takeuchi (1995), denominan el conocimiento explícito. Este se encuentra estructurado y almacenado en la organización, y puede ser distribuido o transmitido fácilmente de un individuo a otro; junto con el conocimiento tácito, que forman parte del sujeto que lo tiene y hace parte de las experiencias personales, no es tangible y su transmisión es más compleja. Pese a la complejidad para identificar el conocimiento tácito, esto puede lograrse mediante estrategias y herramientas que el sujeto construye en el desarrollo de su trabajo y a lo largo de su experiencia profesional e investigativa.

El estudio de Rijnsoever y Hessels (2011), señala que la propensión hacia la colaboración interdisciplinaria se aumenta con la integración de mujeres, miembros que procedan de sectores diferentes al académico o que trabajen en disciplinas estratégicas asociadas a la finalidad del proyecto. De otra parte, es necesario generar cambios en la manera como se retribuye a los colaboradores, pues esto tiende a hacerse con base a indicadores bibliométricos, los cuales sobrevaloran esfuerzos 
disciplinarios mientras que los interdisciplinarios se subestiman.

El desarrollo de proyectos interdisciplinarios genera la necesidad de adoptar estructuras organizacionales, en las que se contemplen integrantes con funciones como: líderes de tareas o áreas de conocimiento, coordinador, equipo de presentación de informes, director, panel de revisión científica independiente y comité directivo. En caso de considerar aspectos transdisciplinarios, es necesario tener personas encargadas del relacionamiento con los interesados del proyecto (Bark, Kragt \& Robson, 2016). Según lo evidenciado por estos autores, el equipo encargado de los informes cumple un rol fundamental para romper barreras que impiden la integración en proyectos interdisciplinarios.

La definición de perfiles de un equipo puede incorporar el criterio de actitud transdisciplinaria (Augsburg, 2005), la cual está relacionada con características tales como: aceptación de diferentes niveles de realidad, apertura a otros puntos de vista, toma de riesgos, disposición para transgredir límites y aprender e investigar de manera creativa. Asimismo, Carr et al. (2018), identifican la flexibilidad, el compromiso y la paciencia, como elementos que influyen en la selección de personas para un trabajo interdisciplinario.

Las instituciones pueden favorecer la colaboración en los equipos, a través de 5 tipos de intervención desde sus directivos (Bark et al., 2016): acordar modelo conceptual, incorporar revisores independientes, apoyar miembros encargados de generar síntesis, fomentar comunicación dentro del proyecto y sistematizar aprendizajes.

Experiencias como las señaladas por Carr et al. (2018), proponen: trabajar en oficinas compartidas con entornos propicios para la interacción, vínculos a través de los programas académicos, realización de seminarios y simposios en los que varía el público invitado, y reuniones entre investigadores con temas afines. A escala de universidad, se pueden generar institutos que agrupen varias facultades o gerenciar proyectos con estructuras tipo matriz (Bark et al., 2016). No obstante, es necesario tener presente que en organizaciones con estructuras "tipo matriz", los empleados pueden terminar trabajando para varios gerentes, lo cual puede convertirse en una desventaja debido a los niveles de ansiedad y estrés en el equipo.

Al momento de iniciar un proyecto interdisciplinar es clave desarrollar un "modelo conceptual preliminar", a través del cual se puedan alinear expectativas y objetivos, revelar diferencias potenciales en puntos de vista o valores entre los participantes, e identificar brechas en el conocimiento Bark et al. (2016). El desarrollo de dicho modelo no requiere la presencia de todo el equipo ni del cliente, basta contar con el líder del proyecto y el equipo encargado de los informes; sin embargo, durante el desarrollo es esencial que se favorezca una buena comunicación, de tal manera que se garantice que los miembros del equipo conozcan las tareas y los plazos que se tracen y se suscriban, sean realistas. Así, el equipo puede ser pragmático en sus ambiciones de investigación disciplinaria, permitiendo que los miembros se adapten y mejoren los resultados de la investigación interdisciplinaria (Bark et al., 2016)

En este sentido, el líder del proyecto debe reunirse con todo el equipo para explicar los plazos y el propósito de la investigación, lo cual contribuye en la alineación de la expectativa en la meta y la aceptación a nivel de equipo. Así mismo, debe comunicarse con todo el equipo de manera periódica, actualizándolos sobre el progreso del proyecto. Al finalizar los proyectos, se deben sistematizar lecciones aprendidas para evaluar los logros obtenidos y reflexionar acerca de los errores; no hacerlo implica perder posibilidades de aprendizaje, que podrían contribuir en el desarrollo de otros proyectos y ayudar al crecimiento de la organización. Si bien la movilidad de los individuos, tanto al interior como fuera de una organización, genera la oportunidad de transmitir lecciones aprendidas, la organización debe contar 
con estrategias para que los aprendizajes no se queden solamente en las personas (Bark et al., 2016).

Aún cuando los aprendizajes se sistematicen y las organizaciones se configuren para facilitar el desarrollo de proyectos interdisciplinares, lograr una integración exitosa requiere un cambio cultural, en el que investigadores, métodos y conceptos de diferentes disciplinas, obtengan un status equivalente a su potencial para contribuir en la resolución de problemas (Bark et al., 2016).

\subsection{Evaluación de la interdisciplinariedad}

Desde hace varias décadas se vienen desarrollando investigaciones interdisciplinares, pero hay pocas referencias enfocadas en la evaluación de las mismas. Tradicionalmente, los resultados de proyectos interdisciplinarios se han medido por indicadores como: i) diversidad de revistas en las que un investigador ha publicado; ii) integración exitosa del conocimiento y comprensión a través de la creación de nuevos campos o disciplinas; o iii) producción de nuevos conocimientos, la calidad y cantidad de los mismos medidos por publicaciones, subvenciones, premios y citas (Carr et al., 2018). Pese a que hacer una evaluación por resultados arroje elementos importantes, es necesario indagar más acerca del cómo están surgiendo estos resultados, cuáles son los factores que respaldan su desarrollo y cómo se puede aumentar su calidad y cantidad.

Bark et al. (2016), sugieren que las organizaciones construyan un marco transparente y consistente, a través del cual se puedan evaluar proyectos, tanto en su fase de propuesta como posterior a su finalización. Entre los aspectos que se pueden incorporar en dicho marco, se encuentran las siguientes preguntas: ¿Incluye la propuesta un modelo conceptual que establezca cómo se conectan los diversos componentes del proyecto y cómo se integrarán? ¿La propuesta evidencia un amplio conocimiento de la literatura relevante en múltiples campos disciplinarios? ¿Se refleja esto en la gama de disciplinas de las que se extraen las referencias? ¿El marco de gestión de proyectos propuesto considera el tiempo extra y la sobrecarga de actividades requeridas para una investigación interdisciplinaria?.

Carr et al. (2018), por su parte, proponen tres categorías a partir de las cuales se puede evaluar el trabajo interdisciplinario: procesos, resultados intermedios intangibles y resultados de investigación y educación tangibles, ver figura 2 . Si bien la consulta de referentes constituye un recurso importante al momento de evaluar un proyecto interdisciplinario, es fundamental indagar por las características propias de cada institución, las cuales varían y afectan la colaboración en cada una de ellas de manera particular (Rijnsoever \& Hessels, 2011). 


\begin{tabular}{|l|}
\multicolumn{1}{c|}{ Procesos } \\
\hline Aprendizaje individual: \\
. Aprender sobre nuevos campos de investigación \\
. Aprender acerca de diferencias y limitaciones entre disciplinas \\
. Identificar colaboradores \\
. Aprender a comunicarse \\
\hline Desarrollo de prácticas de inv. interdisciplinarias: \\
. Clarificar \\
. Aprovechar la diferencia y gerenciar posibles conflictos \\
. Establecer límites \\
Facilitación de la interacción entre investigadores con \\
diferentes visiones del mundo y contextos: \\
. Favorecer la interacción cara a cara \\
. Enlazar personas (conexiones de intermediación)
\end{tabular}

\begin{tabular}{|c|c|}
\hline Resultados intermedios & $\begin{array}{c}\text { Resultados de } \\
\text { investigación y educación }\end{array}$ \\
\hline $\begin{array}{l}\text { Habilidades para } \\
\text { comunicarse, trabajar en } \\
\text { equipo y ampliar la } \\
\text { perspectiva }\end{array}$ & $\begin{array}{l}\text { Nuevo conocimiento } \\
\text { Calidad del trabajo } \\
\text { interdisciplinario }\end{array}$ \\
\hline $\begin{array}{l}\text { Confianza } \\
\text { Conectividad entre } \\
\text { investigadores } \\
\text { Identidad y valores } \\
\text { compartidos }\end{array}$ & . Habilidades profesionales \\
\hline
\end{tabular}

Figura 2. Categorías de evaluación del trabajo interdisciplinar (adaptado de Carr et al., 2018).

\subsection{Método}

Una vez identificados los aspectos claves en el desarrollo de proyectos interdisciplinarios, se realizó un estudio de tipo exploratorio y descriptivo, para caracterizar el conocimiento implícito y explícito de los docentes mediante una entrevista estructurada y encuesta en línea. La población muestreada corresponde a 22 docentes de tiempo completo, que integraron la Facultad de Artes Integradas (FAI), en el año 2018. A continuación se establece la metodología del estudio. i) Caracterización del equipo de trabajo: a través de registros institucionales, se identifica el conocimiento explícito del equipo, tomando como referencia las definiciones de Nonaka y Takeuchi (1995), que de manera directa equivale a la formación de los docentes a nivel de pregrado y posgrado. Estos datos se organizaron bajo las áreas de conocimiento establecidas por la OCDE (Colciencias, 2018a), ver tabla 1. La agrupación de datos de los docentes, permite identificar las áreas de conocimiento predominantes en el equipo de trabajo.

Tabla 1. Conocimiento explícito en la FAl.

\begin{tabular}{|c|c|c|c|c|c|}
\hline & Pregrado & Posgrado & $\begin{array}{l}\text { Área de } \\
\text { conocimiento } \\
\text { pregrado }\end{array}$ & $\begin{array}{l}\text { Gran área de } \\
\text { conocimiento } \\
\text { Posgrado }\end{array}$ & $\begin{array}{l}\text { Área de } \\
\text { conocimiento } \\
\text { Posgrado }\end{array}$ \\
\hline Profesor 1 & Arquitectura & $\begin{array}{l}\text { Magíster en } \\
\ldots\end{array}$ & Humanidades & Ing. y Tecnología & $\begin{array}{l}\text { Ingeniería } \\
\text { ambiental }\end{array}$ \\
\hline Profesor 2 & $\begin{array}{l}\text { Diseño } \\
\text { Industrial }\end{array}$ & & Humanidades & Ing. y Tecnología & $\begin{array}{l}\text { Otras Ingenierías y } \\
\text { Tecnologías }\end{array}$ \\
\hline Profesor 3 & Artes plásticas & & Humanidades & Humanidades & Arte \\
\hline Profesor 4 & Ingeniería & & $\begin{array}{l}\text { Ingeniería y } \\
\text { Tecn. }\end{array}$ & Ingeniería y Tecn. & $\begin{array}{l}\text { Otras Ingenierías y } \\
\text { Tecnologías }\end{array}$ \\
\hline Profesor "n" & $\ldots$ & $\ldots$ & $\ldots$ & $\ldots$ & $\ldots$ \\
\hline
\end{tabular}

ii) Entrevista estructurada: Con el fin de identificar actitudes interdisciplinarias y evidenciar la colaboración entre equipos, esto es, el conocimiento implícito, se aplica una entrevista a cinco profesores que han realizado proyectos interdisciplinarios de la FAI en los últimos 5 años, enfocada en un proyecto en particular. A través de esta se buscaba que los docentes contaran las experiencias 
de trabajo, guiados por preguntas relacionadas con el proyecto, tales como: tiempo de duración, disciplinas participantes, objetivos, la participación de comunidades o empresas, así como, aprendizajes, lecciones aprendidas y las estrategias identificadas para la resolución de conflictos en equipos de trabajo. Este instrumento evidencia la capacidad de articulación con otras disciplinas, los tiempos requeridos para la ejecución de proyectos interdisciplinarios y las posibilidades de mejora. iii) Encuesta en línea: enviada a los 22 profesores y realizada a través de un formulario online, donde se identificaba la persona que la diligenciaba. Los datos recogidos se relacionan con la interacción entre los miembros del equipo de la $\mathrm{FAl}$, en proyectos desarrollados previamente. Así mismo, se indagó acerca de los intereses de vinculación o trabajo entre los profesores, a partir de los temas de interés planteados por cada uno de ellos. En la Tabla 2 se presentan las categorías de las encuestas en términos de interacción entre profesores.

Tabla 2. Categorías de interacción entre profesores a través de la encuesta.

\begin{tabular}{lll}
\hline & $\begin{array}{l}\text { \# profesores con que } \\
\text { quisiera trabajar }\end{array}$ & $\begin{array}{l}\text { \# profesores con que } \\
\text { ha trabajado }\end{array}$ \\
\hline Profesor 1 & 17 & 4 \\
Profesor " $n$ " & $\ldots$ & $\ldots$ \\
\hline
\end{tabular}

Para poder asociar los temas de interés de cada profesor con las áreas de conocimiento en las que se pueden categorizar, se valoraron intereses y enfoques que han tenido en proyectos pasados (Tabla 3). Esta información permitió analizar tendencias de trabajo del equipo en función de las áreas de conocimiento, diferentes a las áreas de formación expuestas previamente como conocimiento explícito; así fue como se identificaron los temas de interés de los docentes, por medio de un análisis de coincidencia de palabras.

Tabla 3. Asociación de temas de interés a áreas de conocimiento.

\begin{tabular}{lll}
\hline & Temas de interés & $\begin{array}{l}\text { Áreas de conocimiento } \\
\text { asociadas al tema }\end{array}$ \\
\hline Profesor 1 & $\begin{array}{l}\text { Gestión de la } \\
\text { Innovación }\end{array}$ & $\begin{array}{l}\text { Ciencias Sociales; Ingeniería; } \\
\text { Humanidades }\end{array}$ \\
Profesor " $n$ " & $\ldots$ & $\ldots$ \\
\hline
\end{tabular}

\section{Resultados y discusión}

\subsection{Caracterización del equipo de trabajo}

La FAl tiene 20 años desde su creación, iniciando labores en el año de 1998. A continuación, se presentan en retrospectiva algunos aspectos que interesan para la presente investigación:

i) Programas pregrado: Arquitectura (20 años) y Diseño Industrial (15 años), ambos en modalidad presencial. ii) Programas posgrado: Maestría en Bioclimática (3 años) y Maestría en Creatividad (6 meses), ambos en modalidad presencial.

iii) Docentes a la fecha: 22 docentes de tiempo completo de los cuales 16 tienen título de maestría, 2 de doctorado y 1 de especialización; durante la realización de esta investigación, habían 3 docentes que estaban llevando a cabo estudios de doctorado y 1 de maestría.

Se tabularon los datos de formación del equipo de profesores de la FAl, identificando la distribución de estos en función de las áreas del conocimiento (Figura 3a). En la clasificación del conocimiento 
a nivel de pregrado, se encontró que el área más explícita son las "Humanidades", porque la formación está centrada en las profesiones de las Artes: a nivel de posgrado cambia su distribución, evidenciando una mirada a otras áreas, como "Ciencias Sociales" e "Ingeniería y Tecnología".

\subsection{Entrevista estructurada}

Se realizó una entrevista estructurada a miembros del equipo que han trabajado en proyectos con otras instituciones, cuyos resultados se sintetizan en la Tabla 4.

Tabla 4. Síntesis de respuestas de la entrevista.

\section{Categorías \\ Interacción con áreas de conocimiento \\ Tiempo de ejecución \\ Motivaciones para vincularse al proyecto}

Objetivo del proyecto Generación proyecto Interacción comunidad

Aprendizajes obtenidos que pueden servirle a la FAI

Retroalimentación del producto final

Discusión y documentación de lecciones aprendidas

Principales problemas proyectos interdisciplinarios resolución de problemas

\section{Respuestas}

Mayor cantidad de interacciones se han dado en "Ingeniería y Tecnología" y "Humanidades"; en menor medida, "Ciencias Sociales" a través de Psicología

Proyectos en su mayoría tardan entre 2 y 4 años

Diversificación de actividades que complementen docencia (investigación, proyección y actuación profesional)

Generación de vínculos con el entorno, exploración de temas de interés y fortalecer el discurso

Interactuar con la comunidad y ayudar a otras personas

Todos los objetivos tienen aplicaciones prácticas, y su impacto va más allá del entorno académico.

Todos los proyectos se generan a partir de contactos por fuera de la FAI

Comunidad siempre participó en conjunto con equipo técnico. Espacios trascendieron las instalaciones de la universidad

Construcción e implementación de metodologías para proyectos interdisciplinarios e interinstitucionales

El valor de la humildad y el darse permiso para explorar nuevos contextos, como alternativa para superar endogamia intelectual y encontrar actividades apasionantes

Enriquecimiento de docencia e investigación a partir de lo desarrollado

Aumento en producción gracias a la diversidad y cantidad de productos generados

En la medida en que aumenta la duración del proyecto, se dificulta más el poder retroalimentar el producto final desde las diferentes disciplinas.

La mayoría de los proyectos no considera este proceso dentro de su programación. En ocasiones se hace, pero de manera informal entre algunos integrantes y no se socializa o dejan evidencias para la FAI

Aptitudes y actitudes para el trabajo en equipo y relacionamiento por fuera de la profesión de origen

Asuntos propios de la gestión de proyectos (tiempo, costo, alcance, seguimiento) Inconsistencia entre funciones y tiempo asignado, impide que se cumplan las metas trazadas Implementar estrategias para gestionar "Proyección Social" e "Investigación" a escala de facultad

Contemplar gestiones asociadas a proyectos interdisciplinares en asignación de tiempos. Incluir retroalimentación y cierre

El proyecto debe ser asumido por perfiles disciplinares adecuados, evitando conflictos de interés 
Los resultados de la entrevista evidencian que la FAl ha trabajado en equipos interdisciplinarios y con diferentes instituciones, permitiendo potenciar las capacidades de creación de los estudiantes y docentes de sus programas. Para hacerlo, se ha valido de eventos, concursos, investigaciones exploratorias y proyectos de extensión, que se han desarrollado bajo las prácticas y experiencias de los docentes; sin embargo, estos procesos y proyectos no han permeado a toda la comunidad académica y no existe una clara documentación de lo realizado.

\subsection{Encuesta en línea}

De los 22 profesores a los que se les envió la encuesta, 19 de ellos la respondieron para un $86,4 \%$ de participación. En la relación de variables

a)

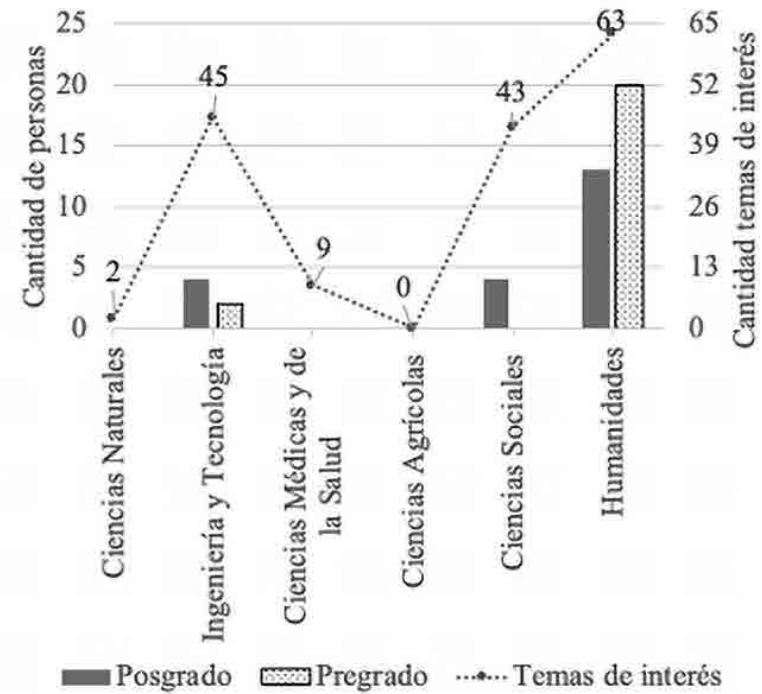

se obtuvo que los profesores en promedio quieren desarrollar proyectos con alrededor de 9 integrantes, con una tendencia a que la mayor cantidad esté por debajo de este promedio (7 y 5 personas). En los extremos se identifican profesores que quieren interactuar con todos y los que menos, quieren hacerlo con mínimo 3 personas (Figura 3b). Con relación a experiencias pasadas, se encontró que en promedio cada profesor ha interactuado con alrededor de 3 personas en proyectos. Quienes más interacciones han tenido alcanzan un valor de 9 personas y 1 no lo ha hecho nunca. En la presente investigación se asumió de manera tácita que todos los investigadores están interesados en el trabajo interdisciplinar y esto puede no ser cierto para algunas personas. Por tanto, se considera necesario indagar por este aspecto en estudios futuros.

b)

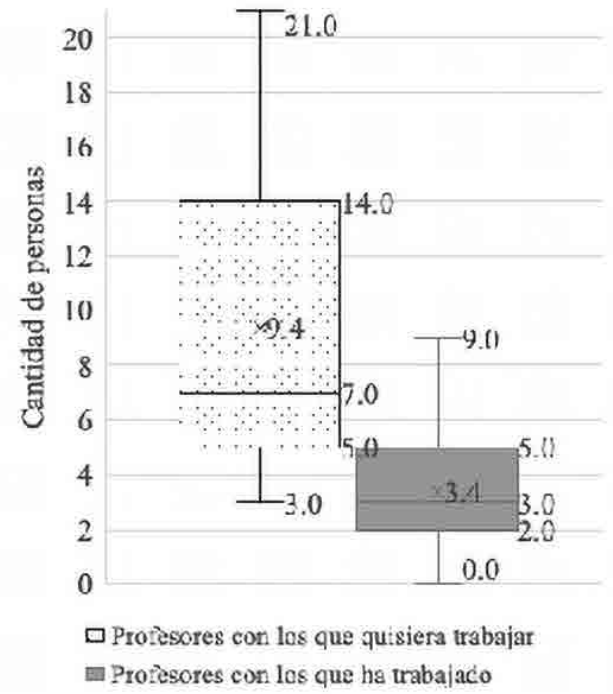

Figura 3. Resultados encuesta: a) Formación e intereses de profesores según áreas de conocimiento; b) Cantidad de profesores con los que quisiera trabajar y con los que ha trabajado.

Un ejercicio similar a futuro debe anunciar claramente que los resultados son confidenciales y los productos que se deriven de esto, nunca señalarán nombres concretos y solamente servirán para 
sacar estadísticas, tal como se hizo en la presente investigación. En esa misma línea, sería recomendable que quienes realicen un estudio de este tipo, no hagan parte del equipo de trabajo sobre el que se está investigando, con el fin de controlar posibles alteraciones en las respuestas de algunos participantes.

A partir de observaciones por parte de los autores del presente artículo, se identifica que, en los procesos cotidianos al interior de la FAl, prima el trabajo independiente o en equipos de 2 a 3 personas. Así mismo, se observa que la conformación de equipos se da en función de la cercanía y afinidad de cada persona. Lo anterior está en consonancia con lo encontrado en la encuesta, en donde la mayor cantidad de docentes ha desarrollado proyectos con 2 o 3 miembros del equipo.

Al cruzar las áreas de formación con los temas de interés distribuidos según áreas del conocimiento, se encuentra que existe correspondencia entre los mismos, ver figura 3a. El área de conocimiento donde se identifica el mayor interés por generar proyectos está representada por "Humanidades" (63 temas), seguida de "Ingeniería y Tecnología" (45) y "Ciencias Sociales" (43). Como áreas potenciales de generación de proyectos interdisciplinares, se identifican las "Ciencias Médicas y de la Salud" y las "Ciencias Naturales"; lo anterior se soporta en el interés que hay en algunos profesores (9 y 2 temas respectivamente) y la ausencia de personas formadas en las mismas al interior de la FAI.

De otra parte, se hizo un análisis de coincidencia de palabras, en las que se encontró que las que más se repiten son: diseño (14 veces), sostenibilidad (6), arquitectura (5) y materiales (4). A partir de esto se evidencia que los intereses de los profesores están enfocados, en su mayoría, en temas propios de las disciplinas del Diseño y la Arquitectura.

\subsection{Estrategias de dinamización}

Pese a las posibilidades que se derivan del trabajo interdisciplinar, las dinámicas del día a día en la FAl, las dificultades para conectar la comunidad académica y la falta de espacios para interactuar, impiden que se capitalicen los aprendizajes obtenidos en experiencias pasadas, lo cual está en consonancia con lo señalado por Bark et al. (2016). En las conversaciones suscitadas a partir de la realización de las encuestas, se evidenció que hay profesores que desconocen lo que hacen algunos integrantes del equipo. Conviene suscitar discusiones en torno al adjetivo que acompaña el nombre de la facultad, planteando los retos, las posibilidades e implicaciones de llamarse "Facultad de Artes Integradas". Los errores cometidos que se pueden evitar, lecciones aprendidas, estrategias exitosas, procedimientos que se deben tener presentes, terminan siendo útiles solamente para las personas que participaron de dichos ejercicios, mientras que la comunidad no obtiene ningún beneficio de lo que se hace. Así mismo, la conclusión en los ciclos de formación o la no continuidad de los docentes en la facultad, hace que la experiencia lograda dentro de la FAI se pierda.

Cuando los programas académicos y los profesionales de una determinada área permanecen encerrados en sí mismos, están perdiéndose oportunidades valiosas de aprendizaje y crecimiento que posibilita el trabajo con otras disciplinas. Para tal fin, como lo proponen Rijnsoever y Hessels (2011), es necesario organizar el conocimiento explícito en relación con las prácticas y ejercicios de los docentes y estudiantes; así como reconocer el conocimiento tácito que se da en las prácticas, herramientas y aproximaciones para el desarrollo de los ejercicios interdisciplinares, que permiten ampliar los marcos de conocimiento.

Igualmente, es necesario que la FAI trabaje en políticas y estrategias que permitan un cambio de mentalidad, a través de aprendizajes desde la perspectiva del trabajo interdisciplinario y metodologías que ayuden a compartir, participar 
y colaborar en el desarrollo de proyectos, abandonando las prevenciones y miedos. Este proceso de gestión del conocimiento requiere incluir entre sus propósitos, la superación de enemistades, preconceptos, malentendidos y experiencias pasadas conflictivas entre los diferentes programas académicos y al interior de estos.

Teniendo presente la importancia de interactuar con comunidades reales, como sucedió en los proyectos sobre los que se realizó la entrevista, es conveniente poner en discusión el impacto que pueden tener sobre el contexto real ciertos tipos de concursos. Dependiendo del planteamiento de los mismos, los requerimientos de perfiles dentro del equipo y los criterios de juzgamiento, las propuestas que se obtienen pueden estar dominadas por la endogamia intelectual de una sola área del conocimiento y carecer de un principio de realidad, disminuyendo las posibilidades de resolver las problemáticas que se trabajan.

El hecho de que en la mayoría de los casos sea mayor la cantidad de profesores con la que se quisiera trabajar (9.4 en promedio), que con los profesores que se ha trabajado (3.4 en promedio), indica que existe la posibilidad de generar interacciones que hasta el momento no se han explorado. Para lograrlo, es necesario implementar estrategias que permitan que dichas voluntades se encuentren y generen proyectos conjuntos, lo cual requiere que se construya confianza entre los miembros del equipo y se generen canales de encuentro.

Una posibilidad para enfocar la actuación de los proyectos de la FAl, consiste en reconocer y orientarse hacia los Objetivos de Desarrollo Sostenible (ODS), identificados y asumidos por diversos gobiernos a nivel mundial, a través de la Organización de las Naciones Unidas (ONU, s.f). Hacerlo, aumenta las probabilidades de encontrar profesionales de diferentes áreas del conocimiento, interesados en resolver las mismas problemáticas; al mismo tiempo, viabiliza las posibilidades de financiación procedentes de diferentes fuentes para iniciativas en esa línea, como lo indica el Departamento Nacional de Planeación (DNP, 2018).

Entre los 17 ODS que existen, en Colombia se realizó un ejercicio de análisis participativo entre la ciudadanía y la comunidad científica, que permitió su priorización para determinar la política pública (Colciencias, 2018b). Basados en este insumo, se realizó un ejercicio de reflexión sobre las temáticas mas relevantes de los ODS para la FAl, estos son: educación de calidad; trabajo decente y crecimiento económico; industria, Innovación e Infraestructura; ciudades y comunidades sostenibles; producción y consumo responsables; y acción por el clima. Otras alternativas están enfocadas en la construcción de un plan estratégico colaborativo y participativo, que permita enfocar al equipo en la visión de la FAl, para la dinamización del conocimiento en pro de objetivos para el trabajo interdisciplinario y el fortalecimiento del mapa de conocimiento.

Así mismo, para la FAl es importante revisar modelos de relación Universidad - Empresa Estado (U-E-E) que incentiven el trabajo interdisciplinario. En el año 2018, se empezó con la revisión de la metodología y los logros alcanzados por el programa: Proyectos de Plicación Profesional (PAP), de la Universidad Jesuita de Guadalajara (ITESO, 2016). Este referente propone un modelo interesante de vinculación U-E-E, con las necesidades de la sociedad y la articulación con el sector empresarial.

\section{Conclusiones}

Se debería fortalecer el trabajo interdisciplinario a través de la integración de otras áreas del conocimiento, que permitan tener miradas sistémicas en los proyectos. Para esto se deben construir espacios de interacción, en los cuales se comparta, debata, complemente y discutan los diferentes puntos de vista del equipo. En la investigación se identificó que hay un potencial de interacción entre los miembros para el desarrollo de proyectos; sin 
embargo, hacen falta estrategias que dinamicen el conocimiento en la Facultad de Artes Integradas (FAl), para la generación de nuevos proyectos.

En este sentido, es importante un cambio en la cultura de trabajo del equipo, en el que se propicien espacios para que la gente se relaje y comparta sus conocimientos, inquietudes $y$ experiencias, con el fin de que puedan generarse nuevas dinámicas de trabajo.

La participación en proyectos de investigación y proyección social de los profesores, aporta en la docencia a la formación de profesionales responsables socialmente. Al mismo tiempo imprime otras dinámicas en el aula de clase, las cuales permiten estar en contacto con el contexto para la aplicación de conocimientos. En este sentido, es necesario que la FAI aclare su visión, de tal manera que sus profesores puedan identificarse con la misma y de esta manera contribuir con su conocimiento al desarrollo colectivo, teniendo claras no solo las funciones sino también las posibilidades de proyección.

\section{Agradecimientos}

A la Universidad de San Buenaventura, Sede Medellín, por la financiación del proyecto 449011-18.02-0012.

\section{Referencias}

Augsburg, T. (2005). Becoming interdisciplinary: an introduction to interdisciplinary studies. New York, USA.

Bark, R. H., Kragt, M. E., \& Robson, B. J. (2016). Evaluating an interdisciplinary research project: Lessons learned for organisations, researchers and funders. International Journal of Project Management, 34 (8), 1449-1459. doi: https://doi. org/10.1016/j.ijproman.2016.08.004
Carr, G., Loucks, D. P., \& Blöschl, G. (2018). Gaining insight into interdisciplinary research and education programmes: A framework for evaluation. Research Policy, 47 (1), 35-48. doi: https://doi. org/10.1016/j.respol.2017.09.010

Colciencias. (2018a). Reconocimiento de Investigadores del Sistema Nacional de Ciencia, Tecnología e Innovación, 233. doi: https://doi.org/10.1017/ CBO9781107415324.004

Colciencias. (2018b). Política Nacional de Ciencia e Innovación para el Desarrollo Sostenible. Bogotá, Colombia: Panamericana Formas e Impresos S.A.

Departamento Nacional de Planeación, DNP. (2018). La Agenda 2030 en Colombia. Recuperado de: https://www.ods.gov.co/

Doucet, I., \& Janssens, N. (2011). Transdisciplinary knowledge production in architecture and urbanism: towards hybrid modes of inquiry. New York, USA: Dordricht.

Francisco I. (2015). Carta Encíclica Laudato Si' del Santo Padre Francisco sobre el cuidado de la casa común. Roma, Italia: Librería Editrice Vaticana.

Galeano-Barrera, C. J., Bellón-Monsalve, D., Zabala-Vargas, S. A., Romero-Riaño, E., \& DuroNovoa, V. (2018). Identificación de los pilares que direccionan a una institución universitaria hacia un Smart-Campus. Revista de Investigación, Desarrollo e Innovación, 9 (1), 127-145. doi: https://doi. org/10.19053/20278306.v9.n1.2018.8511

ITESO. (2016). Proyectos de Aplicación Profesional, PAP, ITESO Aniversario 10. Universidad Jesuita de Guadalajara. Recuperado de: https://www. youtube.com/watch?v=6og0TB9NUR4

Kuhn, T. S. (2004). La Estructura de las Revoluciones Científicas. Buenos Aires, Argentina: Fondo de Cultura Económica. 
Maturana, H., \& Varela, F. (2003). El Árbol del Conocimiento. Las bases biológicas del entendimiento humano. Buenos Aires, Argentina: Editorial Lumen.

Morin, E. (2007). Introducción al Pensamiento Complejo. Barcelona, España: Editorial Gedisa.

Nonaka, I., \& Takeuchi, H. (1995). The knowledge creating company: how Japanese companies create the dynamics of innovation. New York, USA: Oxford University Press.

Organización de las Naciones Unidas, ONU. (s.f.). Objetivos y metas de desarrollo sostenible. Recuperado de: https://www. un.org/sustainabledevelopment/es/ objetivos-de-desarrollo-sostenible/

Organisation for Economic Cooperation and Development, OECD. (1972). Interdisciplinary: Problems of Teaching and Research in Universities. París, Francia: Centre for Educational Research and Innovation.

Rijnsoever, F. J., \& Hessels, L. K. (2011). Factors associated with disciplinary and interdisciplinary research collaboration. Research Policy, 40 (3), 463-472. doi: https://doi.org/10.1016/j. respol.2010.11.001

Thackara, J. (2012). Diseñando para un mundo complejo. México: Designio.

Thompson, J. (2014). Discourses of transdisciplinarity: Looking Back to the Future. Futures, 63, 68-74. doi: https://doi.org/10.1016/j. futures.2015.01.003 